\title{
GENERATING FUNCTIONS FOR A SPECIAL CLASS OF PERMUTATIONS
}

\section{CARLITZ}

ABSTRACT. The paper contains a simple, direct derivation of the generating functions for a class of permutations generalizing the up-down permutations.

1. A permutation $\left(a_{1}, a_{2}, \ldots, a_{n}\right)$ of $Z_{n}=\{1,2, \ldots, n\}$ is called an up-down permutation if

$$
a_{1}<a_{2}, a_{2}>a_{3}, a_{3}<a_{4}, a_{4}>a_{5}, \cdots
$$

This can be represented graphically by

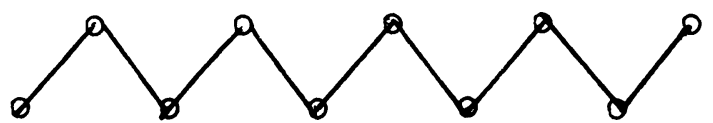

Let $A(n)$ denote the number of up-down permutations of $Z_{n}$. It is well known (see for example [3, pp. 105-112]) that

$$
\sum_{n=0}^{\infty} A(n) \frac{x^{n}}{n !}=\sec x+\tan x \quad(A(0)=A(1)=1) .
$$

This result can be generalized in the following way. Let $k, t$ be fixed integers, $k \geq 2, t \geq 0$. Consider permutations of $Z_{k n+t}$ of the following type:

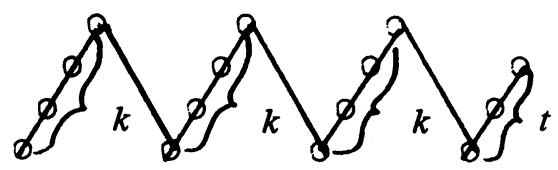

Each incline (except possibly the last) contains $k$ nodes; the last contains $t$ nodes. In the above illustration, $n=3$. For brevity we may call the permutations of the form $(1.3)$, with arbitrary $n,(k, t)$-permutations.

Received by the editors March 28, 1973.

AMS (MOS) subject classifications (1970). Primary 05A15.

${ }^{1}$ Supported in part by NSF grant GP-17031. 
Let $A_{k, t}(n k+t)$ denote the number of such permutations of $Z_{k n+t}$. It is convenient to define

$$
\begin{aligned}
& A_{k, t}(t)=1, \\
& A_{k, t}(s)=0 \quad(0 \leq s<t) .
\end{aligned}
$$

The writer [2] has proved by specializing a general result that

$$
\begin{gathered}
\sum_{n=0}^{\infty} A_{k, 0}(k n) \frac{x^{k n}}{(k n) !}=\frac{1}{\phi_{k, 0}(x)}, \\
\sum_{n=0}^{\infty} A_{k, t}(k n+t) \frac{x^{k n+t}}{(k n+t) !}=\frac{\phi_{k, t}(x)}{\phi_{k, 0}(x)} \quad(t \geq 1),
\end{gathered}
$$

where

The functions $\phi_{k, t}(x)$ defined by $(1.7)$ are the so-called Olivier functions [4]. For some arithmetic properties of the Olivier functions see [1].

For $k=2$, it is clear that (1.5) and (1.6) reduce to (1.2).

The object of the present note is to give a simple self-contained proof of (1.5) and (1.6).

2. We first set up a recurrence for the enumerant $A_{k, t}(k n+t)$. To do this, we consider the effect of removing the element $k n+t$ from a typical $(k, t)$-permutation of $Z_{k n+t^{*}}$ Unless this element is at the extreme right, the given permutation breaks into two pieces: the left-hand piece is a $(k, k-1)$ o permutation, while the right-hand piece is a $(k, t)$-permutation. If $t=1$, it is clear that the extreme right-hand element cannot be $k n+t$. If $t>1$ and $k n+t$ is in the extreme right-hand position, then clearly its removal leaves a $(k, t-1)$-permutation. We accordingly obtain the following recurrences.

$$
A_{k, 0}(k n)=\sum_{j=1}^{n}\left(\begin{array}{c}
k n-1 \\
k j-1
\end{array}\right) A_{k, k-1}(k j-1) A_{k, 0}(k(n-j)),
$$

$$
A_{k, 1}(k n+1)=\sum_{j=1}^{n}\left(\begin{array}{c}
k n \\
k j-1
\end{array}\right) A_{k, k-1}(k j-1) A_{k, 1}(k(n-j)+1),
$$




$$
\begin{aligned}
A_{k, t}(k n+t)= & \sum_{j=1}^{n}\left(\begin{array}{c}
k n+t-1 \\
k j-1
\end{array}\right) A_{k, k-1}(k j-1) A_{k, t}(k(n-j)+t) \\
& +A_{k, t-1}(k n+t-1) \quad(t>1) .
\end{aligned}
$$

Now put

$$
F_{k, t}(x)=\sum_{n=0}^{\infty} A_{k, t}(k n+t) \frac{x^{k n+t}}{(k n+t) !} \quad(t=0,1,2, \ldots) .
$$

For $t=0$, it follows from (2.1) that

$$
\begin{aligned}
F_{k, 0}^{\prime}(x) & =\sum_{n=1}^{\infty} A_{k, 0}(k n) \frac{x^{k n-1}}{(k n-1) !} \\
& =\sum_{n=1}^{\infty} \frac{x^{k n-1}}{(k n-1) !} \sum_{j=1}^{n}\left(\begin{array}{c}
k n-1 \\
k j-1
\end{array}\right) A_{k, k-1}(k j-1) A_{k, 0}(k(n-j)) \\
& =\sum_{j=1}^{\infty} A_{k, k-1}(k j-1) \frac{x^{k j-1}}{(k j-1) !} \sum_{n=0}^{\infty} A_{k, 0}(k n) \frac{x^{k n}}{(k n) !}
\end{aligned}
$$

Hence we get

$$
F_{k, 0}^{\prime}(x)=F_{k, k-1}(x) F_{k, 0}(x) .
$$

Next, for $t=1$, it follows from (2.2) that

$$
\begin{aligned}
F_{k, 1}^{\prime}(x) & =\sum_{n=0}^{\infty} A_{k, 1}(k n+1) \frac{x^{k n}}{(k n) !} \\
& =1+\sum_{n=1}^{\infty} \frac{x^{k n}}{(k n) !} \sum_{j=1}^{n}\left(\begin{array}{c}
k n \\
k j-1
\end{array}\right) A_{k, k-1}(k j-1) A_{k, 1}(k(n-j)+1) \\
& =1+\sum_{j=1}^{\infty} A_{k, k-1}(k j-1) \frac{x^{k j-1}}{(k j-1) !} \sum_{n=0}^{\infty} A_{k, 1}(k n+1) \frac{x^{k n+1}}{(k n+1) !}
\end{aligned}
$$

Thus

$$
F_{k, 1}^{\prime}(x)=1+F_{k, k-1}(x) F_{k, 1}(x) .
$$

Finally, for $t>1$, we have 


$$
\begin{aligned}
F_{k, t}^{\prime}(x)= & \sum_{n=0}^{\infty} A_{k, t}(k n+t) \frac{x^{k n+t-1}}{(k n+t-1) !} \\
= & A_{k, t}(t) \frac{x^{t-1}}{(t-1) !} \\
& +\sum_{n=1}^{\infty} \frac{x^{k n+t-1}}{(k n+t-1) !}\left\{\sum_{j=1}^{n}\left(\begin{array}{c}
k n+t-1 \\
k j-1
\end{array}\right) A_{k, k-1}(k j-1) A_{k, t}(k(n-j)+t)\right. \\
= & \left.\sum_{n=0}^{\infty} A_{k, t-1}(k n+t-1) \frac{x^{k n+t-1}}{(k n+t-1) !}+A_{k, t-1}(k n+t-1)\right\} \\
& +\sum_{j=1}^{\infty} A_{k, k-1}(k j-1) \frac{x^{k j-1}}{(k j-1) !} \sum_{n=0}^{\infty} A_{k, t}(k n+t) \frac{x^{k n+t}}{(k n+t) !} \cdot
\end{aligned}
$$

Therefore

$$
F_{k, t}^{\prime}(x)=F_{k, t-1}(x)+F_{k, k-1}(x) F_{k, t}(x) \quad(t>1)
$$

3. In order to solve the system (2.5), (2.6), (2.7) for the $F_{k, t}(x)$ we put

$$
\begin{gathered}
F_{k, 0}(x)=1 / \psi_{k, 0}(x), \\
F_{k, t}(x)=\psi_{k, t}(x) / \psi_{k, 0}(x) \quad(t>0) .
\end{gathered}
$$

Since the series defining $F_{k, 0}(x)$ has constant term equal to 1 , it is clear that $\psi_{k, 0}(x), \psi_{k, t}(x)$ are well defined.

Substituting from (3.1) and (3.2) in (2.5), we get

$$
-\frac{\psi_{k, 0}^{\prime}(x)}{\psi_{k, 0}^{2}(x)}=\frac{\psi_{k, k-1}(x)}{\psi_{k, 0}(x)} \frac{1}{\psi_{k, 0}(x)}
$$

so that

$$
\psi_{k, 0}^{\prime}(x)=-\psi_{k, k-1}(x)
$$

Similarly, it follows from (2.6) that

$$
\frac{\psi_{k, 0}(x) \psi_{k, 1}^{\prime}}{\psi_{k, 0}^{2}(x)} \frac{\psi_{k, 0}^{\prime}(x) \psi_{k, 1}(x)}{\psi_{k, 0}(x)}=1+\frac{\psi_{k, k-1}(x) \psi_{k, 1}(x)}{\psi_{k}^{2}}
$$


Making use of (3.3), this reduces to

$$
\psi_{k, 1}^{\prime}(x)=\psi_{k, 0}(x)
$$

In exactly the same way, it follows from (2.7) and (3.3) that

$$
\psi_{k, t}^{\prime}(x)=\psi_{k, t-1}(x) \quad(t>1) .
$$

Combining (3.4) and (3.5) we have

$$
\psi_{k, t}^{\prime}(x)=\psi_{k, t-1}(x) \quad(t \geq 1)
$$

It follows at once from (3.3) and (3.6) that

$$
d^{k} \psi_{k, 0}(x) / d x^{k}=-\psi_{k, 0}(x) .
$$

It is clear from (3.1) and (2.4) that

$$
\psi_{k, 0}(x)=\sum_{n=0}^{\infty} a_{n} \frac{x^{k n}}{(k n) !} \quad\left(a_{0}=1\right) .
$$

Thus

$$
\frac{d^{k}}{d x^{k}} \psi_{k, 0}(x)=\sum_{n=0}^{\infty} a_{n+1} \frac{x^{k n}}{(k n) !} .
$$

Hence by (3.7), $a_{n+1}=-a_{n}$, so that $a_{n}=(-1)^{n}$. It follows that

$$
\psi_{k, 0}(x)=\sum_{n=0}^{\infty}(-1)^{n} \frac{x^{k n}}{(k n) !}
$$

Applying (3.3) we get

$$
\psi_{k, k-1}(x)=\sum_{n=0}^{\infty}(-1)^{n} \frac{x^{k n+k-1}}{(k n+k-1) !} .
$$

Repeated application of (3.6) now gives

$$
\psi_{k, t}(x)=\sum_{n=0}^{\infty}(-1)^{n} \frac{x^{k n+t}}{(k n+t) !} \quad(0 \leq t<k) .
$$

To remove the restriction on $t$ in (3.10), we note first that, by (3.2),

$$
\psi_{k, t}(x)=\sum_{n=0}^{\infty} a_{n, t} \frac{x^{k n+t}}{(k n+t) !} \quad(t \geq 0) .
$$


In view of $(3.6)$ we have

$$
a_{n, t}=a_{n, t-1} \quad(t \geq 1) .
$$

Since, in particular, $a_{n, k-1}=(-1)^{n}$, it follows from (3.11) that $a_{n, t}=$ $(-1)^{n}(t \geq k)$. Therefore

$$
\psi_{k, t}(x)=\sum_{n=0}^{\infty}(-1)^{n} \frac{x^{k n+t}}{(k n+t) !} \quad(t \geq 0) .
$$

Comparing (3.12) with (1.7) we get

$$
\psi_{k, t}(x)=\phi_{k, t}(x) \quad(t \geq 0)
$$

This evidently completes the proof of (1.5) and (1.6).

\section{REFERENCES}

1. L. Carlitz, Some arithmetic properties of the Olivier functions, Math. Ann. 128 (1955), 412-419. MR 16, 677.

2. - Permutations with prescribed pattern, Math. Nachr. 58 (1973), 31-53.

3. E. Netto, Lehrbuch der Combinatorik, Teubner, Leipzig, 1927.

4. L. Olivier, Bemerkungen über eine Art von Funktionen, welche Eigenschaften haben, wie die Cosinus und Sinus, J. Reine Angew. Math. 2 (1827), 243-251.

DEPAR TMENT OF MATHEMATICS, DUKE UNIVERSITY, DURHAM, NORTH CAROLINA 27706 